\title{
Establishing an integrated health research training program at Dalhousie University
}

I ncreasingly, health professionals work in teams to deliver high-quality heath care, education and research. Within the Faculties of Medicine, Health Professions and Dentistry, there are a number of trainee groups that represent essentially all aspects of medical research, clinical care and educational sectors. As a result of the evolving nature of research and clinical care, today's trainees will be functioning as part of multidisciplinary teams in an environment influenced by rapid changes in research capacity (new technologies) and political and societal pressures. There will be unprecedented opportunities to translate new knowledge into meaningful strategies to manage disease and promote health.

The Faculties of Medicine, Health Professions and Dentistry at Dalhousie University are in the process of establishing a number of new support programs to ensure that our trainees are equipped to excel in translating discoveries into disease diagnosis and management. The Associate Dean of Research at the Faculty of Medicine has recently appointed two Assistant Deans representing the areas of Graduate and Postdoctoral Studies and Research-Clinical Departments. The Faculty of Dentistry has also recently appointed an Assistant Dean of Research. The five Associate/Assistant Deans from the Health Faculties meet regularly to better align resources, as well as research and educational mandates. As a result, the three Faculties are organizing and overseeing an Integrated Health Research Training Program to serve the needs of all trainees. The program will feature learning sessions to develop skills in writing grants and proposals, making oral and written presentations, establishing a research operation, and building effective collaborations. The program will also provide insights into managing the difficult balance among professional activities (teaching, research, clinical care, administration), and provide information on alternate careers including those in policy, patent law, technology transfer, and the pharmaceutical industry. This type of institutional support program is unique in the country, and as the Integrated Health Research Training Program is established, it will put Nova Scotia in a favoured position for recruitment and retention of the highest quality trainees.

\section{Gerald C. Johnston and}

Christopher R. McMaster

Office of the Dean, Medical Research Services Faculty of Medicine, Dalhousie University

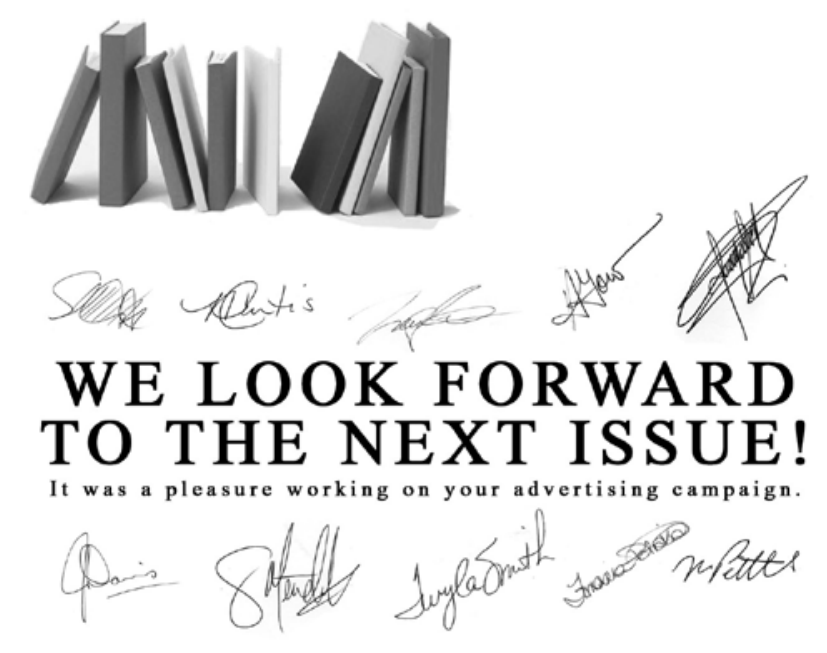

Want to know how we make other Universities and College publications look great?

Contact us to find out how 1.866 .362 .3331 w w w. cu-ads.com

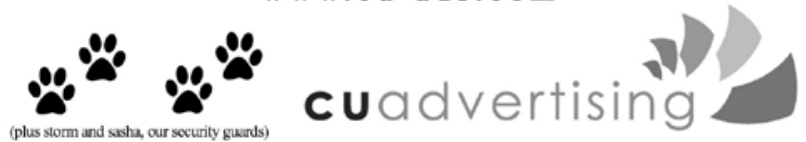

